\title{
Imaging of Apoptosis: The Need to Distinguish Tracer Uptake Rate from Regional Contribution of Blood Flow
}

\author{
Hans-Jürgen Machulla \\ Institute for Radiopharmacy, Eberhard Karls University of Tuebingen, Tuebingen, Germany
}

$\mathbf{I}$ oncology, PET is known to contribute substantially to localization of tumors and metastases and to control of therapy. One particularly attractive application is the assay of apoptosis induced by chemotherapy or radiation. Various tracers, such as ${ }^{99 \mathrm{~m}} \mathrm{Tc}-$ annexin- $\mathrm{V}$ and ${ }^{18} \mathrm{~F}-\mathrm{ML}-10$, are under discussion and in application. At Stanford University, the group of Jianghong Rao reported the unique concept of a radiotracer that reaches its final form within the apoptotic cells (1). This happens in a series of 3 reaction steps: the ${ }^{18} \mathrm{~F}$-labeled precursor, ${ }^{18} \mathrm{~F}$-C-SNAT, is selectively cleaved by caspase- $3 / 7$ between the $\mathrm{D}$ and $\mathrm{X}$ of the DEVD-X peptide sequence (where $\mathrm{X}=$ any amino acid and DEVD = Asp-Glu-Val-Asp) and the disulfide is reduced to a thiol by intracellular glutathione; the released amino and thiol groups react with the cyano group on the hydroxyquinoline at the other end of the molecule via an intramolecular reaction; and the resulting cyclized compound (cyclic $\left.{ }^{18} \mathrm{~F}-\mathrm{C}-\mathrm{SNAT}\right)$ is highly lipophilic and thus remains trapped within the apoptotic cell and apoptotic bodies.

\section{See page 1415}

It is noteworthy that in organic chemistry, intramolecular reactions are known to be up to $10^{4}$ times faster than the corresponding intermolecular ones. Rao's group reported a half-life of $119.8 \mathrm{~s}$ for that type of reaction. Therefore, the intermediates formed after cleavage are unlikely to react with other molecules within the cellular environment, and cyclic ${ }^{18} \mathrm{~F}-\mathrm{C}-\mathrm{SNAT}$ can be assumed to be the only ${ }^{18} \mathrm{~F}$ product.

Biologic evaluations validated, in a proof of concept, that ${ }^{18} \mathrm{~F}$ C-SNAT is a tracer for the assessment of apoptosis. A parallel study with a fluorescence scaffold, C-SNAF, demonstrated the same concept on a molecular level (2). Overall, the concept is well suited to tracing apoptotic cells resulting from chemotherapy.

In this issue of The Journal of Nuclear Medicine, Rao's group continues its studies of ${ }^{18} \mathrm{~F}-\mathrm{C}$-SNAT in tumor-bearing mice with and without doxorubicin treatment and applies a pharmacokinetic analysis using a 2-tissue-compartment model (3). As the input function, blood activity was acquired from the images by placing

Received May 6, 2015; revision accepted May 12, 2015.

For correspondence or reprints contact: Hans-Jürgen Machulla, University

of Tuebingen, Roentgenweg 15, Tuebingen D-72076, Germany.

E-mail: machulla@uni-tuebingen.de

Published online May 29, 2015.

COPYRIGHT (C) 2015 by the Society of Nuclear Medicine and Molecular Imaging, Inc.

DOI: $10.2967 /$ jnumed.115.159285 a region of interest over the heart, as can conveniently be done in small animals. The compartment modeling allowed the investigators to distinguish tracer accumulation in apoptotic tissue from blood activity within the same region of interest. $K_{1}$ and $k_{3}$ are the velocity constants for tracer delivery from blood and accumulation in tissue, respectively. In tumors after treatment with doxorubicin, $k_{3}$ changed from $0.03 \pm 0.01$ to $0.12 \pm 0.02 \mathrm{~min}^{-1}$. The velocity constants increased by a factor of 4 , whereas the direct calculations (based on injected dose of activity) predicted a factor of 1.4 $(1.17 \pm 0.17$ vs. $0.81 \pm 0.28$ percentage injected dose [\% ID]/g) (1). In muscle, $k_{3}$ is close to zero, as is expected because of the biochemical concept of ${ }^{18} \mathrm{~F}-\mathrm{C}$-SNAT. If uptake is related to injected dose, $0.7 \pm 0.1 \% \mathrm{ID} / \mathrm{g}$ is found in muscle of untreated mice, and a corresponding $1.6 \pm 0.2 \% \mathrm{ID} / \mathrm{g}$ is found in tumor tissue. Thus, determination of the accumulation rate $k_{3}$ clearly estimates the treatment outcome.

Independently of tracer accumulation in tissue, the 2-tissuecompartment model allows for the determination of $K_{1}$, which describes tracer delivery from blood. After treatment with doxorubicin, $K_{1}$ increased from $0.31 \pm 0.05$ to $1.03 \pm 0.14 \mathrm{~g} / \mathrm{mL} \times \min$. In muscle, the delivery constant also increased, from $0.17 \pm 0.03$ to $1.03 \pm 0.1 \mathrm{~g} / \mathrm{mL} \times \mathrm{min}$. In tumor and muscle tissue, increases in $K_{1}$ are thought to result from a systemic effect originating with doxorubicin, as has been described for ${ }^{18}$ F-FDG (4-6).

In experimental tracer applications, data are usually calculated as $\% \mathrm{ID} / \mathrm{g}$. Because such uptake values represent primarily blood activity, reference regions are applied so that blood activity can be subtracted. The investigators' work illustrates that tracer accumulation and blood activity are parameters independent of each other and may change independently. In their work, $k_{3}$ is zero in muscle, but a high $K_{1}$ would feign tracer accumulation if uptake were determined as $\% \mathrm{ID} / \mathrm{g}$.

The pharmacokinetic modeling allows for discrimination between tracer accumulation in tissue and tracer activity in blood. The study presented in this issue of JNM (3) clearly shows ${ }^{18} \mathrm{~F}-\mathrm{C}$ SNAT to be a tracer very well suited to clinical application. It is particularly noteworthy that the applied modeling can be used directly in clinical studies. For the radiosynthesis of ${ }^{18} \mathrm{~F}-\mathrm{C}$-SNAT, Rao's group applied the tracer principle. ${ }^{18} \mathrm{~F}-\mathrm{C}-\mathrm{SNAT}$ was prepared with high efficiency, labeling precursor was removed, and the amount of peptide could be kept low (1). Therefore, clinical translation can easily be realized by the microdosing concept $(<100 \mu \mathrm{g})$, for which the necessary toxicology data can be determined quickly and inexpensively.

Over the last 10 years, pharmacokinetic analyses have somewhat fallen away from the main focus of the community, but the work of Rao's group impressively shows the usefulness of applyingand even more, the need to apply-2-tissue-compartment modeling 
to PET data. Today, PET systems are fast enough to perform dynamic registrations, and a subroutine can analyze data resulting in parametric imaging of regional $k_{3}$ values so that modeling can be used even in daily PET work. PET/MR imaging opens the technical possibility of having blood flow recorded simultaneously with tracer registration. In February 2015, the $4^{\text {th }}$ Tübingen PET/MR Workshop revealed the need for simultaneous blood flow measurements, for which a technical implementation into clinical PET/MR imaging systems was discussed to soon be forthcoming.

In summary, the work of Rao's group brings ${ }^{18} \mathrm{~F}-\mathrm{C}$-SNAT close to direct translation on the clinical level-a perspective that is truly promising. The tracer is well suited to PET assessment of apoptosis caused by chemotherapy. Even more, the work exhibits an importance far beyond the development of ${ }^{18} \mathrm{~F}-\mathrm{C}$ SNAT, as the value of pharmacokinetic modeling lies in its ability to determine tracer accumulation in tissue independently of blood flow. This work clearly illustrates how the way has been paved for precisely performed PET follow-up studies of therapies and disease progression.

\section{DISCLOSURE}

No potential conflict of interest relevant to this article was reported.

\section{REFERENCES}

1. Shen B, Jeon J, Palner M, et al. Positron emission tomography imaging of druginduced tumor apoptosis with a caspase-triggered nanoaggregation probe. Angew Chem Int Ed Engl. 2013;52:10511-10514.

2. Ye D, Shuhendler AJ, Cui L, et al. Bioorthogonal cyclization-mediated in situ selfassembly of small-molecule probes for imaging caspase activity in vivo. Nat Chem. 2014;6:519-526.

3. Palner M, Shen B, Jeon J, Lin J, Chin FT, Rao J. Preclinical kinetic analysis of the caspase-3/7 PET tracer ${ }^{18} \mathrm{~F}-\mathrm{C}-\mathrm{SNAT}$ : quantifying the changes in blood flow and tumor retention after chemotherapy. J Nucl Med. 2015;56:1415-1421.

4. Mankoff DA, Dunnwald LK, Gralow JR, et al. Changes in blood flow and metabolism in locally advanced breast cancer treated with neoadjuvant chemotherapy. $\mathrm{J} \mathrm{Nucl} \mathrm{Med}$. 2003;44:1806-1814.

5. Tseng J, Dunnwald LK, Schubert EK, et al. ${ }^{18}$ F-FDG kinetics in locally advanced breast cancer: correlation with tumor blood flow and changes in response to neoadjuvant chemotherapy. J Nucl Med. 2004;45:1829-1837.

6. Røe K, Aleksandersen TB, Kristian A, et al. Preclinical dynamic ${ }^{18}$ F-FDG PET: tumor characterization and radiotherapy response assessment by kinetic compartment analysis. Acta Oncol. 2010;49:914-921. 